\title{
NATURAL NUMEROSITIES OF SETS OF TUPLES
}

\author{
MARCO FORTI AND GIUSEPPE MORANA ROCCASALVO
}

\begin{abstract}
We consider a notion of "numerosity" for sets of tuples of natural numbers that satisfies the five common notions of Euclid's Elements, so it can agree with cardinality only for finite sets. By suitably axiomatizing such a notion, we show that, contrasting to cardinal arithmetic, the natural "Cantorian" definitions of order relation and arithmetical operations provide a very good algebraic structure. In fact, numerosities can be taken as the non-negative part of a discretely ordered ring, namely the quotient of a formal power series ring modulo a suitable ("gauge") ideal. In particular, special numerosities, called "natural", can be identified with the semiring of hypernatural numbers of appropriate ultrapowers of $\mathbb{N}$.
\end{abstract}

\section{INTRODUCTION}

In this paper we consider a notion of "equinumerosity" on sets of tuples of natural numbers, i.e. an equivalence relation, finer than equicardinality, that satisfies the celebrated five Euclidean common notions about magnitudines ([8]), including the principle that "the whole is greater than the part". This notion preserves the basic properties of equipotency for finite sets. In particular, the natural Cantorian definitions endow the corresponding numerosities with a structure of a discretely ordered semiring, where 0 is the size of the emptyset, 1 is the size of every singleton, and greater numerosity corresponds to supersets.

This idea of "Euclidean" numerosity has been recently investigated by V. Benci, M. Di Nasso and M. Forti in a sequel of papers, starting with [1] (see also [3]). In particular, in [2] the size of arbitrary sets (of ordinals) was approximated by means of directed unions of finite sets, while in [7] finite dimensional point sets over the real line, and in [4 entire "mathematical universes" are considered.

Here we focus on sets of tuples of natural numbers, and we show that the existence of equinumerosity relations for such sets is equivalent to the existence of a class of prime ideals, which we call "gauge ideals", of a special ring of formal power series in countably many indeterminates. As in all papers quoted above, special ultrafilters are used in order to model numerosities. In fact, the gauge ideals corresponding to the equinumerosities called "natural" are in biunique correspondence with special ultrafilters over the set $\mathbb{N}<\omega$ of all finite subsets of $\mathbb{N}$. These ultrafilters, also called "gauge", may be of independent interest, as their connection with other classes of special ultrafilters considered in the literature is not clear. In fact, we only prove here that all selective ultrafilters are gauge.

The paper is organized as follows. In Section 1 we derive from the Euclidean common notions a few set theoretic principles that are the basis of our formal

Received by the editors June 18, 2012 and, in revised form, December 2, 2012.

2010 Mathematics Subject Classification. Primary 03E65, 03F25; Secondary 03A05, 03C20.

The first author's research was partially supported by MIUR Grant PRIN 2009, Italy. 
definition of equinumerosity relation. Then we prove that the corresponding numerosities form an ordered semiring that can be obtained as the non-negative part of the quotient of a ring $\mathcal{R}$ of formal power series in countably many indeterminates, modulo suitable prime ideals. In Section 2 we show how numerosities can be embedded in rings of hyperreal numbers obtained by means of special ultrafilters. In particular, all "natural" numerosities are essentially non-standard natural numbers. Actually, they arise through isomorphisms with ultrapowers $\mathbb{N}_{\mathcal{N}}^{\mathbb{N}}$ modulo "gauge ultrafilters". A few final remarks and open questions are contained in Section 3.

In general, we refer to [6] for definitions and facts concerning ultrapowers, ultrafilters, and non-standard models that are used in this paper.

The authors are grateful to Andreas Blass and Mauro Di Nasso for useful discussions.

\section{EQUinUmerosity OF POINT SETS}

In this section we study a notion of "numerosity" for point sets of natural numbers, i.e. for sets of tuples of natural numbers. This numerosity will be defined by starting from an equivalence relation of "equinumerosity", denoted by $\approx$, that satisfies the basic properties of equipotency between finite sets.

In particular, we assume first that equinumerosity satisfies the following 1

AP (Aristotelian Principle): $A \approx B$ if and only if $A \backslash B \approx B \backslash A$.

The axiom AP is a compact equivalent set theoretic formulation of the second and third common notions of Euclid's Elements: "If equals be added to equals, the wholes are equal", and "if equals be subtracted from equals, the remainders are equal". (A precise statement of this equivalence is given in Proposition 1.7 below.) On the other hand, for infinite sets, the Aristotelian Principle is clearly incompatible with the Cantorian notion of equicardinality.

Notice that the first common notion "things which are equal to the same thing are also equal to one another" is already secured by the assumption that equinumerosity is an equivalence relation.

Together with the notion of "having the same numerosity", it is natural to introduce a "comparison of numerosities", so as to satisfy the fifth Euclidean common notion "the whole is greater than the part". Of course, this comparison must be coherent with equinumerosity, so we are led to the following

Definition. We say that $A$ is greater than $B$, denoted by $A \succ B$, or equivalently that $B$ is smaller than $A$, denoted by $B \prec A$, if there exist $A^{\prime}, B^{\prime}$ such that $A^{\prime} \supset B^{\prime}, A \approx A^{\prime}$, and $B \approx B^{\prime}$. The natural idea that numerosities of sets are always comparable, combined with the fifth Euclidean notion, gives the following trichotomy property:

ZP (Zermelian Principle): Exactly one of the following three conditions holds:
(a) $A \approx B$;
(b) $A \succ B$;
(c) $A \prec B$.

1 We call this principle "Aristotelian", because the favourite example of "axiom" quoted by Aristotle is "if equals be subtracted from equals, the remainders are equal." 
We shall see below that the Zermelian Principle implies that given two sets one is equinumerous to some superset of the other, and obviously that no proper subset is equinumerous to the set itself. (See Proposition 1.4)

We stress that both properties AP and ZP hold for equipotency between finite sets, while both fail badly for equipotency between infinite sets. So we cannot assume that equipotent sets are always equinumerous, but we have to give a suitable interpretation of the fourth Euclidean common notion "things applying [exactly] onto one another are equal to one another" 2 We choose two kinds of numerosity preserving bijections, namely "permutations of components" and "rising dimension":

TP (Transformation Principle): If $T$ is $1-1$, and $T(a)=\left(a_{\tau 1}, \ldots, a_{\tau k}\right)$ is a permutation of $a=\left(a_{1}, \ldots, a_{k}\right)$ for all $a \in A$, then $A \approx T[A]$.

UP (Unit Principle): $A \times\{n\} \approx A$ for all $n \in \mathbb{N}$.

Remark that the Unit Principle cannot be consistently assumed for all point sets $A$. In fact, if $A=\{n,(n, n),(n, n, n), \ldots\}$, then $A \times\{n\}$ is a proper subset of $A$, and so it cannot be equinumerous to $A$. So we have to restrict the principle UP. In view of further developements, in particular in order to obtain a semiring of numerosities, a convenient choice is the family of all "finitary" point sets, i.e. sets that contain only finitely many tuples for each finite set of components. Denote by

$$
\mathbb{W}=\left\{A \subseteq \mathcal{P}\left(\bigcup_{k \in \mathbb{N}} \mathbb{N}^{k}\right) \mid \forall n \exists h \forall k>h\left(A \cap\{0, \ldots, n\}^{k}=\emptyset\right)\right\}
$$

the family of all finitary point sets. Remark that $\mathbb{W}$ is a proper superset of the family of all "finite dimensional" point sets $\mathbb{W}_{0}=\bigcup_{d \in \mathbb{N}} \mathcal{P}\left(\bigcup_{k=1}^{d} \mathbb{N}^{k}\right)$ that has been considered in [5, 9].

Finally, in order to define a product of numerosities, we could introduce the following principle:

PP (Product Principle): If $A \approx A^{\prime}$ and $B \approx B^{\prime}$, then $A \times B \approx A^{\prime} \times B^{\prime}$.

In order to make $\mathbb{W}$ closed under Cartesian products, we follow the usual practice, and we identify Cartesian products with the corresponding "concatenations". That is, for every $A \subseteq \mathbb{N}^{k}$ and for every $B \subseteq \mathbb{N}^{h}$, we identify

$$
A \times B=\left\{\left(\left(a_{1}, \ldots, a_{k}\right),\left(b_{1}, \ldots, b_{h}\right)\right) \mid\left(a_{1}, \ldots, a_{k}\right) \in A,\left(b_{1}, \ldots, b_{h}\right) \in B\right\}
$$

with

$$
A \times B=\left\{\left(a_{1}, \ldots, a_{k}, b_{1}, \ldots, b_{h}\right) \mid\left(a_{1}, \ldots, a_{k}\right) \in A \text { and }\left(b_{1}, \ldots, b_{h}\right) \in B\right\} .
$$

With this convention, we have that $A \times\left\{x_{1}, x_{2}, \ldots, x_{k}\right\}=A \times\left\{x_{1}\right\} \times\left\{x_{2}\right\} \times$ $\ldots \times\left\{x_{k}\right\}$, and so, using also the Transformation Principle, we obtain the general property

$$
\{P\} \times A \approx A \times\{P\} \approx A \text { for any point } P \in \mathbb{N}^{k} .
$$

In particular, any two singletons are equinumerous.

However, assuming this convention, the Product Principle cannot be consistently postulated in the above formulation for all sets in $\mathbb{W}$, because different pairs of tuples may share the same concatenation. E.g. both $((1,2),(3,4,5))$ and $((1,2,3),(4,5))$ produce $(1,2,3,4,5)$. So one should consider concatenated products as "multisets", where each tuple comes with its (finite) "multiplicity". We

\footnotetext{
${ }^{2}$ See the accurate discussion of this Euclidean common notion by T. Heath in 8 .
} 
prefer to consider only pure sets, so we restrict the Product Principle to suitably defined "multipliable pairs".

Let us call the sets $A, B \in \mathbb{W}$ multipliable if different pairs $(a, b) \in A \times B$ have different concatenations. (For instance, every set of $\mathbb{W}_{0}$ is multipliable with every subset of $\mathbb{N}^{k}$.) We shall restrict PP to products of multipliable sets. We can now give a precise definition of the notion of equinumerosity relation.

Definition 1.1. Let

$$
\mathbb{W}=\left\{A \subseteq \mathcal{P}\left(\bigcup_{k \in \mathbb{N}} \mathbb{N}^{k}\right) \mid \forall n \exists h \forall k>h\left(A \cap\{0, \ldots, n\}^{k}=\emptyset\right)\right\}
$$

be the set of all finitary point sets.

- An equivalence relation $\approx$ on $\mathbb{W}$ is an equinumerosity if the following properties are fullfilled for all $A, B \in \mathbb{W}$ :

AP: $A \approx B$ if and only if $A \backslash B \approx B \backslash A$.

ZP: Exactly one of the following three conditions holds:

$$
\text { either } A \approx B \text {, or } A \succ B \text {, or } A \prec B \text {, }
$$

where $A$ is greater than $B$, denoted by $A \succ B$, or equivalently $B$ is smaller than $A$, denoted by $B \prec A$, if there exist $A^{\prime}, B^{\prime}$ such that

$$
A^{\prime} \supset B^{\prime}, A \approx A^{\prime} \text {, and } B \approx B^{\prime} \text {. }
$$

TP: If $T$ is $1-1$, and $T(a)$ is a permutation of $a$ for all $a \in A$, then $A \approx T[A]$. UP: $A \times\{n\} \approx A$ for all $n \in \mathbb{N}$.

PP: If $A, B$ and $A^{\prime}, B^{\prime}$ are multipliable pairs and $A \approx A^{\prime}, B \approx B^{\prime}$, then

$$
A \times B \approx A^{\prime} \times B^{\prime} .
$$

Definition 1.2. Let $\approx$ be an equinumerosity relation on the set $\mathbb{W}$.

- The numerosity of $A$ (w.r.t. $\approx$ ) is the equivalence class $[A]_{\approx}=\{B \in \mathbb{W} \mid B \approx A\}$ of all point sets equinumerous to $A$, denoted by $\mathfrak{n}_{\approx}(A)$.

- The set of numerosities of $\approx$ is the quotient set $\mathfrak{N}_{\approx}=\mathbb{W} / \approx$, and

- the numerosity function associated to $\approx$ is the canonical map $\mathfrak{n}_{\approx}: \mathbb{W} \rightarrow \mathfrak{N}_{\approx}$.

We drop the subscript $\approx$ whenever the equinumerosity relation is fixed.

Clearly the Unit Principle formalizes the natural idea that singletons have "unitary" numerosity. A trivial but important consequence of this axiom is the existence of infinitely many pairwise disjoint equinumerous copies of any point set. Moreover, infinitely many of them can be taken to be multipliable with any fixed set of $\mathbb{W}$. Namely

Proposition 1.3. Let $A, B \in \mathbb{W}$ be point sets. For $m, n, h, k \in \mathbb{N}$ put

$$
A\left(m^{h}, n^{k}\right)=A \times\{m\}^{h} \times\{n\}^{k} .
$$

Assuming UP, the sets $A\left(m^{h}, n^{k}\right)$ are equinumerous to $A$ for all $h, k$, and are pairwise disjoint, disjoint from $B$, and multipliable with $B$ for all sufficiently large $h, k$.

Proof. The first assertion is obvious. In order to prove the remaining ones, put $p=\max \{m, n\}$ and assume that $A \cap\{0, \ldots, p\}^{l}=\emptyset$ for $l \geq j$. Then $A\left(m^{h}, n^{k}\right)$ is disjoint from $A$ and multipliable with $B$ whenever $h, k \geq j$. Moreover, $A\left(m^{h}, n^{k}\right) \cap$ $A\left(m^{h^{\prime}}, n^{k^{\prime}}\right)=\emptyset$ whenever $h, k, h^{\prime}, k^{\prime} \geq j$ and $k \neq k^{\prime}$.

In the following proposition we list several important properties of the binary relation $\prec$. 


\section{Proposition 1.4.}

(i) $A \prec B$ holds if and only if $B$ is equinumerous to a proper superset $B^{\prime}$ of $A$. Hence, given two sets in $\mathbb{W}$, one is equinumerous to a superset of the other one.

(ii) The relation $\prec$ is a preorder on $\mathbb{W}$ that induces a total ordering on the quotient set $\mathfrak{N}=\mathbb{W} / \approx$.

Proof. ( $i$ ) If there exists a proper superset $B^{\prime}$ of $A$ that is equinumerous to $B$, then, from the definition of $\prec$, we conclude that $A \prec B$. Conversely, suppose that $A \prec B$, that is, there exist sets $A^{\prime}$ and $B^{\prime}$ such that $A^{\prime} \subset B^{\prime}, A \approx A^{\prime}$ and $B \approx B^{\prime}$. By possibly applying Proposition 1.3. we may assume without loss of generality that $A \cap A^{\prime}=A \cap B^{\prime}=\emptyset$. Put $C=B^{\prime} \backslash A^{\prime}$, and consider $A \cup C$, which is a proper superset of $A$. By AP we have

$$
A \cup C \approx B \Longleftrightarrow A \cup C \approx B^{\prime} \Longleftrightarrow A=(A \cup C) \backslash B^{\prime} \approx B^{\prime} \backslash(A \cup C)=A^{\prime} .
$$

So $A \cup C$ is a proper superset of $A$ equinumerous to $B$.

(ii) Clearly $A \nprec A$ because $A$ cannot be equinumerous to a proper superset of itself. In order to prove transitivity of the relation $\prec$, we state the following lemma:

Lemma 1.5. Let $\approx$ be a relation of equinumerosity on $\mathbb{W}$, and let $A \approx B$. Then for each proper superset $A^{\prime}$ of $A$ there exists a proper superset $B^{\prime}$ of $B$ such that $A^{\prime} \approx B^{\prime}$.

Proof. According to $(i)$, let us consider the three possible cases:

(1) $A^{\prime} \approx B$ : then $A^{\prime} \approx A$, which is impossible by ZP.

(2) There exists a proper superset $A^{\prime \prime}$ of $A^{\prime}$ such that $A^{\prime \prime} \approx B$ : then $A$ would be equinumerous to the proper superset $A^{\prime \prime}$, again contradicting ZP.

(3) There exists a proper superset $B^{\prime}$ of $B$ such that $A^{\prime} \approx B^{\prime}$ and the lemma is proved.

Now we can prove transitivity of the relation $\prec$. Assume that $A \prec B$ and $B \prec C$ : then there exist proper supersets $A^{\prime}, B^{\prime}$ of $A, B$ respectively, such that $A^{\prime} \approx B$ and $B^{\prime} \approx C$. Then, by Lemma 1.5, there exists a proper superset $A^{\prime \prime}$ of $A$ such that $A^{\prime \prime} \approx B^{\prime} \approx C$, and so $A \prec C$.

Now we prove that the principle AP is equivalent to the conjunction of the second and third common notions of Euclid, when formalized in the following way:

SP (Sum Principle): Let $A, A^{\prime}, B, B^{\prime} \in \mathbb{W}$ be such that $A \cap B=\emptyset$ and $A^{\prime} \cap B^{\prime}=\emptyset$. If $A \approx A^{\prime}$ and $B \approx B^{\prime}$, then $A \cup B \approx A^{\prime} \cup B^{\prime}$.

DP (Difference Principle): Let $A, A^{\prime}, C, C^{\prime} \in \mathbb{W}$ be such that $A \subseteq C$ and $A^{\prime} \subseteq C^{\prime}$. If $A \approx A^{\prime}$ and $C \approx C^{\prime}$, then $C \backslash A \approx C^{\prime} \backslash A^{\prime}$.

This equivalence has been proved in [9, and also in [5] for a slightly different notion of equinumerosity. We repeat the proof here for convenience of the reader, because we need these facts in the sequel. We begin by stating the following lemma:

Lemma 1.6. Let $\approx$ be an equivalence relation for which $\mathrm{AP}$ holds and let $A, B, A^{\prime}$, $B^{\prime} \in \mathbb{W}$ be such that $B \subseteq A$ and $B^{\prime} \subseteq A^{\prime}$. If $B \approx B^{\prime}$, then

$$
A \backslash B \approx A^{\prime} \backslash B^{\prime} \Longleftrightarrow A \approx A^{\prime} .
$$

Proof. Put $B_{0}=B \backslash A^{\prime}, \quad B_{0}^{\prime}=B^{\prime} \backslash A, \quad C=B \cap B^{\prime}, \quad B_{1}=B \backslash\left(B_{0} \cup C\right)$, $B_{1}^{\prime}=B^{\prime} \backslash\left(B_{0}^{\prime} \cup C\right), C_{0}=A \backslash\left(B \cup A^{\prime}\right), C_{0}^{\prime}=A^{\prime} \backslash\left(B^{\prime} \cup A\right), E=\left(A \cap A^{\prime}\right) \backslash\left(B \cup B^{\prime}\right)$. So we obtain pairwise disjoint sets $B_{0}, B_{0}^{\prime}, C, B_{1}, B_{1}^{\prime}, C_{0}, C_{0}^{\prime}$, E such that $B=B_{0} \cup B_{1} \cup C$, 
$B^{\prime}=B_{0}^{\prime} \cup B_{1}^{\prime} \cup C, A \backslash A^{\prime}=B_{0} \cup C_{0}, \quad A^{\prime} \backslash A=B_{0}^{\prime} \cup C_{0}^{\prime}, \quad A \backslash B=B_{1}^{\prime} \cup C_{0} \cup E$, $A^{\prime} \backslash B^{\prime}=B_{1} \cup C_{0}^{\prime} \cup E$. By $\mathrm{AP}$ we can write

$$
A \approx A^{\prime} \Longleftrightarrow B_{0} \cup C_{0}=A \backslash A^{\prime} \approx A^{\prime} \backslash A=B_{0}^{\prime} \cup C_{0}^{\prime},
$$

$A \backslash B \approx A^{\prime} \backslash B^{\prime} \Longleftrightarrow B_{1}^{\prime} \cup C_{0}=(A \backslash B) \backslash\left(A^{\prime} \backslash B^{\prime}\right) \approx\left(A^{\prime} \backslash B^{\prime}\right) \backslash(A \backslash B)=B_{1} \cup C_{0}^{\prime}$.

By hypothesis and AP we can write

$$
B_{0} \cup B_{1} \cup C=B \approx B^{\prime}=B_{0}^{\prime} \cup B_{1}^{\prime} \cup C \Longrightarrow B_{0} \cup B_{1} \approx B_{0}^{\prime} \cup B_{1}^{\prime},
$$

and hence

$$
B_{0} \cup B_{1} \cup C_{0} \approx B_{0}^{\prime} \cup B_{1}^{\prime} \cup C_{0}
$$

Suppose that $A \backslash B \approx A^{\prime} \backslash B^{\prime}$, that is, $B_{1}^{\prime} \cup C_{0} \approx B_{1} \cup C_{0}^{\prime}$. It follows that $B_{0}^{\prime} \cup B_{1}^{\prime} \cup C_{0} \approx B_{0}^{\prime} \cup B_{1} \cup C_{0}^{\prime}$ and hence $B_{0} \cup B_{1} \cup C_{0} \approx B_{0}^{\prime} \cup B_{1} \cup C_{0}^{\prime}$, and we conclude that $A \approx A^{\prime}$.

Conversely, if $A \approx A^{\prime}$, that is, $B_{0} \cup C_{0} \approx B_{0}^{\prime} \cup C_{0}^{\prime}$, we have $B_{0} \cup B_{1} \cup C_{0} \approx$ $B_{0}^{\prime} \cup B_{1} \cup C_{0}^{\prime}$ and hence $B_{0}^{\prime} \cup B_{1}^{\prime} \cup C_{0} \approx B_{0}^{\prime} \cup B_{1} \cup C_{0}^{\prime}$, and we conclude that $A \backslash A^{\prime} \approx A^{\prime} \backslash A$.

Proposition 1.7. Let $\approx$ be an equivalence relation. The Axiom AP is equivalent to the conjunction of the two principles SP and DP.

Proof. The conjunction of SP and DP yields AP, namely

$$
\begin{gathered}
\left(A \backslash A^{\prime}\right) \cup\left(A \cap A^{\prime}\right)=A \approx A^{\prime}=\left(A^{\prime} \backslash A\right) \cup\left(A \cap A^{\prime}\right) \Longrightarrow^{D P}\left(A \backslash A^{\prime}\right) \approx\left(A^{\prime} \backslash A\right), \\
A \backslash\left(A \cap A^{\prime}\right)=\left(A \backslash A^{\prime}\right) \approx\left(A^{\prime} \backslash A\right)=A^{\prime} \backslash\left(A \cap A^{\prime}\right) \Longrightarrow{ }^{S P} A \approx A^{\prime} .
\end{gathered}
$$

Conversely, assume AP; then, by Lemma 1.6, both SP and DP hold.

We prove now that our notion of equinumerosity satisfies what can be viewed as a necessary condition, videlicet that finite point-sets are equinumerous if and only if they have the same "number of elements".

Proposition 1.8. Let $\approx$ be an equinumerosity relation, and let $A, B \in \mathbb{W}$ be finite sets. Then

$$
A \approx B \Longleftrightarrow|A|=|B| .
$$

Moreover, if $X$ is infinite, then $X \succ A$. Hence $\mathbb{N}$ can be taken as an initial segment of the set of numerosities $\mathfrak{N}$ corresponding to $\approx$.

Proof. First observe that $\emptyset$, being a proper subset of any non-empty set $A$, cannot be equinumerous to $A$.

Secondly, we have already remarked that any two singletons $\{a\},\{b\}$ are equinumerous. Moreover, if $C \approx\{b\}$, then $C$ is a singleton. In fact, let $c$ be an element of $C$; then $\{c\} \approx\{b\} \approx C$ and hence $\{c\}=C$, because $C$ cannot be a proper superset of $\{c\}$.

Finally, given two finite sets $A$ and $B$, we proceed by induction on $k$, the least cardinality of the sets $A, B$. The case $k=1$ has already been dealt with. Assume the thesis true for $k \leq n$ and let $A$ and $B$ be finite sets such that $n+1=\min \{|A|,|B|\}$. Pick $a \in A$ and $b \in B$, and put $A^{\prime}=A \backslash\{a\}, B^{\prime}=B \backslash\{b\}$. Since $\{a\} \approx\{b\}$, Lemma 1.6 and the induction hypothesis yield

$$
A \approx B \Longleftrightarrow A^{\prime} \approx B^{\prime} \Longleftrightarrow\left|A^{\prime}\right|=\left|B^{\prime}\right| \Longleftrightarrow|A|=|B| .
$$

Now if $X$ is an infinite set and $A$ is a finite set, we can find a proper subset $B$ of $X$, such that $|A|=|B|$, so we conclude that $A \prec X$. 
By the above proposition, we can identify each natural number $n \in \mathbb{N}$ with the equivalence class of all those point sets that have finite cardinality $n$.

Starting from the equivalence relation of equipotency, Cantor introduced the algebra of cardinals by means of disjoint unions and Cartesian products. So we could similarly introduce an algebra on "numerosities". The given axioms have been chosen so as to guarantee that numerosities are naturally equipped with a "nice" algebraic structure. (This is to be contrasted with the awkward cardinal algebra, where e.g. $\kappa+\mu=\kappa \cdot \mu=\max \{\kappa, \mu\}$ for all infinite $\kappa, \mu$.)

Theorem 1.9. Let $\mathfrak{N}$ be the set of numerosities of the equinumerosity relation $\approx$. Then there exist unique operations + and $\cdot$, and a unique linear order $<$ on $\mathfrak{N}$, such that for all point sets $X, Y \in \mathbb{W}$ :

(1) $\mathfrak{n}(X)+\mathfrak{n}(Y)=\mathfrak{n}(X \cup Y)$ whenever $X \cap Y=\emptyset$;

(2) $\mathfrak{n}(X) \cdot \mathfrak{n}(Y)=\mathfrak{n}(X \times Y)$ whenever $X, Y$ are multipliable;

(3) $\mathfrak{n}(X)<\mathfrak{n}(Y)$ if and only if $Y \approx Y^{\prime}$ for some proper superset $Y^{\prime} \supset X$.

The resulting structure on $\mathfrak{N}$ is the non-negative part of a discretely ordered ring $(\mathfrak{R}, 0,1,+, \cdot,<)$. Moreover, if the fundamental subring of $\mathfrak{R}$ is identified with $\mathbb{Z}$, then $\mathfrak{n}(X)=|X|$ for every finite point set $X$.

We could prove the above theorem by the very same arguments used in [5] or in 9]. However, we prefer to obtain a more precise algebraic characterization of the arithmetic of numerosities. To this aim, we consider a suitable ring of formal power series with integer coefficients, and we prove that the set of numerosities can be identified with the non-negative part of the quotient of this ring modulo a suitable prime ideal.

- Let $\mathbb{T}=\left\langle t_{n} \mid n \in \mathbb{N}\right\rangle$ be a sequence of indeterminates. Let $\mathbf{A}$ be the set of all eventually zero sequences $\mathbf{a}=\left(a_{0}, a_{1}, \ldots\right)$ of non-negative integers, and denote by $t^{\mathbf{a}}$ the monomial $\prod_{i \in \mathbb{N}} t_{i}^{a_{i}}$.

So any series $S$ in the variables of $\mathbb{T}$ can be written as $S=\sum_{\mathbf{a} \in \mathbf{A}} n_{\mathbf{a}} t^{\mathbf{a}}$, where $n_{\mathbf{a}}$ is the coefficent of the monomial $t^{\mathbf{a}}$.

- Given a point $x=\left(x_{1}, \ldots, x_{d}\right) \in \mathbb{N}^{d}$, consider the sequence a $\in \mathbf{A}$, where $a_{i}=\left|\left\{j \mid x_{j}=i\right\}\right|$ and associate to $x$ the monomial $t_{x}=t^{\mathbf{a}}$.

- The characteristic series of the non-empty point set $X \in \mathbb{W}$ is the formal series

$$
S_{X}=\sum_{x \in X} t_{x}=\sum_{\mathbf{a} \in \mathbf{A}} n_{\mathbf{a}} t^{\mathbf{a}}, \text { where } n_{\mathbf{a}}=\left|\left\{x \in X \mid t_{x}=t^{\mathbf{a}}\right\}\right| .
$$

(If $X=\emptyset$, put $S_{X}=0$.)

- Characteristic series behave well with respect to unions, differences and products:

$$
\begin{gathered}
S_{X}+S_{Y}=S_{X \cup Y}+S_{X \cap Y} \quad, \quad S_{X}-S_{Y}=S_{X \backslash Y} \text { if } Y \subset X, \\
S_{X} \cdot S_{Y}=S_{X \times Y} \text { if } X, Y \text { are multipliable } 3
\end{gathered}
$$

Remark 1.10. A series $S=\sum n_{\mathbf{a}} t^{\mathbf{a}}$ with non-negative integer coefficients is the characteristic series of a set $X \in \mathbb{W}$ if and only if for all $\mathbf{a}=\left(a_{0}, a_{1}, \ldots\right)$ we have $n_{\mathbf{a}} \leq \frac{k !}{\prod a_{i} !}$, where $k=\sum_{i} a_{i}$.

In fact, the number of different sequences $\mathbf{a}=\left(a_{0}, a_{1}, \ldots\right)$ that correspond to the same monomial $t^{\mathbf{a}}$ is $\frac{k !}{\prod a_{i} !}$, where $k=\sum_{i} a_{i}$ is the degree of the monomial.

\footnotetext{
${ }^{3}$ Remark that if the product $X \times Y$ is considered as a multiset where each tuple comes with its multiplicity, then the equality holds for all $X, Y \in \mathbb{W}$.
} 
- Let $\mathcal{R}$ be the ring of all formal series of bounded degree $d_{n}$ in each variable $t_{n} \in \mathbb{T}$ with coefficients $n_{\mathbf{a}}$ such that, for some $b \in \mathbb{N}$,

$$
\left|n_{\mathbf{a}}\right| \leq b \frac{\left(\sum_{i} a_{i}\right) !}{\prod a_{i} !} .
$$

- Let $\mathcal{R}^{+}$be the multiplicative subset of the positive series, i.e. the series in $\mathcal{R}$ having only positive coefficients.

- Let $\mathfrak{I}_{0}$ be the ideal of $\mathcal{R}$ generated by $\left\{t_{n}-1 \mid n \in \mathbb{N}\right\}$.

It is easily seen that $\mathcal{R}$ is the subring with identity of $\mathbb{Z}[[\mathbb{T}]]$ generated by the set of the characteristic series of all point sets. Moreover, every positive series $P \in \mathcal{R}^{+}$ is equivalent modulo $\mathfrak{I}_{0}$ to some characteristic series.

Lemma 1.11. Every positive series $P \in \mathcal{R}^{+}$is equivalent modulo $\mathfrak{I}_{0}$ to the characteristic series of some set in $\mathbb{W}$. So any series $S \in \mathcal{R}$ can be written as $S=$ $S_{X}-S_{Y}+S_{0}$ for suitable $X, Y \in \mathbb{W}$ and $S_{0} \in \mathfrak{I}_{0}$.

Proof. Suppose that the series $S$ has non-negative coefficients $n_{\mathbf{a}} \leq b \frac{k !}{\prod a_{i} !}$, where $k=\sum_{i} a_{i}$. We can decompose $S$ in at most $b$ series with coefficients satisfying the conditions for being characteristic, plus a non-negative integer $a$. So we can write $S=a+S_{X_{1}}+\ldots+S_{X_{s}}$, where $s \leq b$ and for all $i, X_{i} \in \mathbb{W}$. (The sets $X_{i}$ may fail to be distinct, in general.) According to Proposition 1.3, we can multiply the integer $a$ and each series $S_{X_{i}}$ by suitable monomials $t_{m}^{h} t_{n}^{k}$, so that $a t_{m}^{h} t_{n}^{k}$ is the characteristic series of a set $Y_{0}$ of tuples containing $h$ times $m$ and $k$ times $n$, and the remaining series are the characteristic series of pairwise disjoint sets $Y_{i}$ equinumerous to $X_{i}$. Clearly, each $S_{X_{i}}$ is equivalent modulo $\mathfrak{I}_{0}$ to $S_{Y_{i}}$, so putting $Y=Y_{0} \cup Y_{1} \cup \ldots \cup Y_{s}$, we have that $S$ is equivalent modulo $\mathfrak{I}_{0}$ to the characteristic series $S_{Y}=S_{Y_{0}}+\ldots+S_{Y_{s}}$.

The final assertion of the theorem follows by considering separately the positive and the negative parts of any given series of $\mathcal{R}$.

In order to classify all equinumerosities, we introduce the following definition:

Definition 1.12. Call an ideal $\mathfrak{I}$ of $\mathcal{R}$ a gauge ideal if

- $\mathfrak{I}_{0} \subseteq \mathfrak{I}$,

- $\mathcal{R}^{+} \cap \mathfrak{I}=\emptyset$, and

- for all $S \in \mathcal{R} \backslash \mathfrak{I}$ there exists $P \in \mathcal{R}^{+}$such that either $S+P \in \mathfrak{I}$ or $S-P \in \mathfrak{I}$

Remark that when $\mathfrak{I}$ is a gauge ideal the quotient $\mathcal{R} / \mathfrak{I}$ is a discretely ordered ring whose positive elements are the cosets $P+\mathfrak{I}$ for $P \in \mathcal{R}^{+}$. In particular, $\mathfrak{I}$ is a prime ideal of $\mathcal{R}$ that is maximal among the ideals disjoint from $\mathcal{R}^{+}$.

Then we have

Theorem 1.13. There exists a biunique correspondence between equinumerosity relations on the space $\mathbb{W}$ of all point sets over $\mathbb{N}$ and gauge ideals on the ring $\mathcal{R}$ of all bounded power series in countably many indeterminates. In this correspondence, if the equinumerosity $\approx$ corresponds to the ideal $\mathfrak{I}$, then

$$
X \approx Y \Longleftrightarrow S_{X}-S_{Y} \in \mathfrak{I} .
$$

More precisely, let $\mathfrak{n}: \mathbb{W} \rightarrow \mathfrak{N}$ be the numerosity function associated to $\approx$, and let $\pi: \mathcal{R} \rightarrow \mathcal{R} / \mathfrak{I}$ be the canonical projection. Then there exists a unique 
order preserving embedding $j$ of $\mathfrak{N}$ onto the non-negative part of $\mathcal{R} / \mathfrak{I}$ such that the following diagram commutes:

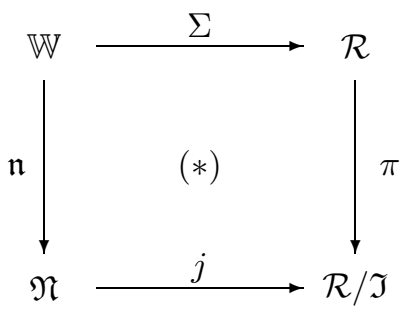

(where $\Sigma$ maps any $X \in \mathbb{W}$ to its characteristic series $S_{X} \in \mathcal{R}^{+}$).

The ordered semiring structure induced on $\mathfrak{N}$ by $j$ satisfies the conditions

(1) $\mathfrak{n}(X)+\mathfrak{n}(Y)=\mathfrak{n}(X \cup Y)$ whenever $X \cap Y=\emptyset$;

(2) $\mathfrak{n}(X) \cdot \mathfrak{n}(Y)=\mathfrak{n}(X \times Y)$ whenever $X, Y$ are multipliable;

(3) $\mathfrak{n}(X)<\mathfrak{n}(Y)$ if and only if $Y \approx Y^{\prime}$ for some proper superset $Y^{\prime} \supset X$.

Proof. Given a gauge ideal $\mathfrak{I}$ over $\mathcal{R}$, we define the equivalence relation over $\mathbb{W}$ by condition (**): $X \approx Y \Longleftrightarrow S_{X}-S_{Y} \in \mathfrak{I}$. We prove that the relation $\approx$ so defined is in fact an equinumerosity relation.

AP: We can write $S_{X}-S_{Y}=S_{X \backslash Y}+S_{X \cap Y}-S_{Y \backslash X}-S_{X \cap Y}=S_{X \backslash Y}-S_{Y \backslash X}$; hence $X \approx Y \Longleftrightarrow X \backslash Y \approx Y \backslash X$.

ZP: Assume first that $S_{X}-S_{Y} \in \mathfrak{I}$, that is, $X \approx Y$. If there exists a superset $Y^{\prime}$ of $X$ such that $S_{Y^{\prime}}-S_{Y} \in \mathfrak{I}$, then $S_{Y^{\prime}}-S_{X}=S_{Y^{\prime} \backslash X} \in \mathfrak{I} \cap \mathcal{R}^{+}$, a contradiction. Similarly there exists no superset $X^{\prime}$ of $Y$ equinumerous to $X$.

Now assume that $S_{X}-S_{Y} \notin \mathfrak{I}$, that is, $X \neq \nsucc$. Then there exists a series $P \in \mathcal{R}^{+}$such that either $S_{X}-S_{Y}+P$ or $S_{X}-S_{Y}-P$ belongs to $\mathfrak{I}$, by the third property of gauge ideals. Assume without loss of generality that $S_{X}-S_{Y}+P \in \mathfrak{I}$. Then $P$ is equivalent modulo $\mathfrak{I}$ to the characteristic series of a set $Z$ that we can choose disjoint from $X$, by Lemma 1.11. Hence $S_{X \cup Z}-S_{Y}=S_{X}-S_{Y}+S_{Z} \in \mathfrak{I}$, whence $(X \cup Z) \approx Y$.

TP: $S_{X}$ is obviously equal to $S_{T[X]}$ whenever $T$ is a transformation that permutes the components of the tuples in $X$. Hence $X \approx T[X]$.

UP: For any set $X \in \mathbb{W}$, we have $S_{X \times\{n\}}-S_{X}=S_{X} \cdot\left(t_{n}-1\right) \in \mathfrak{I}_{0} \subseteq \mathfrak{I}$, i.e. $X \times\{n\} \approx X$.

PP: Let $X, Y$ and $X^{\prime}, Y^{\prime}$ be multipliable point sets. If $S_{X}-S_{X^{\prime}}$ and $S_{Y}-S_{Y^{\prime}}$ belong to $\mathfrak{I}$, that is, $X \approx X^{\prime}$ and $Y \approx Y^{\prime}$, then

$$
S_{X \times Y}-S_{X^{\prime} \times Y^{\prime}}=\left(S_{X}-S_{X^{\prime}}\right) \cdot S_{Y}+S_{X^{\prime}} \cdot\left(S_{Y}-S_{Y^{\prime}}\right) \in \mathfrak{I}
$$

hence $X \times Y \approx X^{\prime} \times Y^{\prime}$.

Conversely, given an equinumerosity relation $\approx$ over $\mathbb{W}$, let $\mathfrak{I}$ be the ideal of $\mathcal{R}$ generated by the set $\left\{S_{X}-S_{Y} \mid X \approx Y\right\} \cup\left\{t_{0}-1\right\}$. We prove that $\mathfrak{I}$ is a gauge ideal.

First observe that the ideal $\mathfrak{I}_{0}$ is also generated by $\left\{t_{n}-t_{0} \mid n \in \mathbb{N}\right\} \cup\left\{t_{0}-1\right\}$, so $\mathfrak{I}_{0}$ is contained in $\mathfrak{I}$, because $t_{n}-t_{0}=S_{\{n\}}-S_{\{0\}}$.

By Lemma 1.11, given a series $S \in \mathcal{R}$ there exist two sets $X$ and $Y$ of $\mathbb{W}$, such that $S$ is equivalent modulo $\mathfrak{I}$ to $S_{X}-S_{Y}$. If $S \notin \mathfrak{I}$, then $X \not \approx Y$, so, without loss of generality, we may assume that there exists a superset $X^{\prime}$ of $Y$ such that $X \approx X^{\prime}$, 
that is, $S_{X}-S_{X^{\prime}} \in \mathfrak{I}$, by ZP. Then we can write $S_{X}-S_{X^{\prime}}=S_{X}-S_{X^{\prime} \backslash Y}-S_{Y} \in \mathfrak{I}$, and hence $S_{X}-S_{Y}$ is congruent modulo $\mathfrak{I}$ to $S_{X^{\prime} \backslash Y} \in \mathcal{R}^{+}$.

It remains to prove that $\mathcal{R}^{+} \cap \mathfrak{I}=\emptyset$. We need the following fact:

Claim. For any $S \in \mathfrak{I}$ there exist $h, k \in \mathbb{N}$ and finitely many (not necessarily distinct) sets $Z_{1}, W_{1}, \ldots, Z_{m}, W_{m}$ of $\mathbb{W}$, such that $Z_{1} \approx W_{1}, \ldots, Z_{m} \approx W_{m}$ and

$$
t_{0}^{h} t_{1}^{k} S=\left(S_{Z_{1}}-S_{W_{1}}\right)+\ldots+\left(S_{Z_{m}}-S_{W_{m}}\right) .
$$

Proof. By definition of $\mathfrak{I}$, there exist $S_{0}, \ldots, S_{n} \in \mathcal{R}$ such that

$$
S=S_{0} \cdot\left(t_{0}-1\right)+S_{1} \cdot\left(S_{X_{1}}-S_{Y_{1}}\right)+\ldots+S_{n} \cdot\left(S_{X_{n}}-S_{Y_{n}}\right),
$$

with $X_{1} \approx Y_{1}, \ldots, X_{n} \approx Y_{n}$.

According to Lemma 1.11, there exist $a_{i} \in \mathbb{Z}$ and $X_{1 i}, \ldots, X_{s i} ; Y_{1 i}, \ldots, Y_{t i} \in \mathbb{W}$ such that

Then we have

$$
S_{i}=a_{i}+S_{X_{1 i}}+\ldots+S_{X_{s i}}-S_{Y_{1 i}}-\ldots-S_{Y_{t i}} .
$$

$$
\begin{aligned}
S_{i} \cdot\left(S_{X_{i}}-S_{Y_{i}}\right)= & a_{i} \cdot\left(S_{X_{i}}-S_{Y_{i}}\right)+S_{X_{1 i}} \cdot\left(S_{X_{i}}-S_{Y_{i}}\right)+\ldots+S_{X_{s i}} \cdot\left(S_{X_{i}}-S_{Y_{i}}\right) \\
& -S_{Y_{1 i}} \cdot\left(S_{X_{i}}-S_{Y_{i}}\right)-\ldots-S_{Y_{t i}} \cdot\left(S_{X_{i}}-S_{Y_{i}}\right) .
\end{aligned}
$$

By Proposition 1.3 we can find $h, k \in \mathbb{N}$ such that all sets

$$
U_{j i}=X_{j i} \times\{0\}^{h} \times\{1\}^{k}, V_{l i}=Y_{l i} \times\{0\}^{h} \times\{1\}^{k}
$$

are multipliable with both $X_{i}$ and $Y_{i}$.

For all $i>0$ we have

$$
\begin{aligned}
t_{0}^{h} t_{1}^{k} S_{i}\left(S_{X_{i}}-S_{Y_{i}}\right)= & a_{i} t_{0}^{h} t_{1}^{k}\left(S_{X_{i}}-S_{Y_{i}}\right)+S_{U_{1 i}}\left(S_{X_{i}}-S_{Y_{i}}\right)+\ldots+S_{U_{s i}}\left(S_{X_{i}}-S_{Y_{i}}\right) \\
& -S_{V_{1 i}}\left(S_{X_{i}}-S_{Y_{i}}\right)-\ldots-S_{V_{t i}}\left(S_{X_{i}}-S_{Y_{i}}\right) \\
= & a_{i}\left(S_{\{0\}^{h} \times\{1\}^{k} \times X_{i}}-S_{\{0\}^{h} \times\{1\}^{k} \times Y_{i}}\right)+\left(S_{U_{1 i} \times X_{i}}-S_{U_{1 i} \times Y_{i}}\right) \\
& +\ldots+\left(S_{U_{s i} \times X_{i}}-S_{U_{s i} \times Y_{i}}\right)-\left(S_{V_{1 i} \times X_{i}}-S_{V_{1 i} \times Y_{i}}\right) \\
& -\ldots-\left(S_{V_{t i} \times X_{i}}-S_{V_{t i} \times Y_{i}}\right) .
\end{aligned}
$$

Similarly, observing that $t_{0}-1=S_{\{0\}}-1$, we obtain for $i=0$

$$
\begin{aligned}
t_{0}^{h} t_{1}^{k} S_{0}\left(t_{0}-1\right)= & a_{0}\left(S_{\{0\}^{h+1} \times\{1\}^{k}}-S_{\{0\}^{h} \times\{1\}^{k}}\right)+\left(S_{U_{10} \times\{0\}}-S_{U_{10}}\right) \\
& +\ldots+\left(S_{U_{s 0} \times\{0\}}-S_{U_{s 0}}\right)-\left(S_{V_{10} \times\{0\}}-S_{V_{10}}\right) \\
& -\ldots-\left(S_{V_{t 0} \times\{0\}}-S_{V_{t 0}}\right) .
\end{aligned}
$$

(If $a_{0}>0$ we take $a_{0}$ times the difference $S_{\{0\}^{h+1} \times\{1\}^{k}}-S_{\{0\}^{h} \times\{1\}^{k}}$, whereas if $a_{0}<0$ we take $-a_{0}$ times the difference $S_{\{0\}^{h} \times\{1\}^{k}}-S_{\{0\}^{h+1} \times\{1\}^{k}}$.)

By PP we have $U_{j i} \times X_{i} \approx U_{j i} \times Y_{i}$ and $V_{j i} \times X_{i} \approx V_{j i} \times Y_{i}$ for all $j$, and obviously $\{0\}^{h} \times\{1\}^{k} \times X_{i} \approx\{0\}^{h} \times\{1\}^{k} \times Y_{i}$. Hence, by taking $Z_{1}, \ldots, Z_{m}$ and $W_{1}, \ldots, W_{m}$ to be an enumeration of the sets $U_{j i} \times X_{i}, V_{j i} \times X_{i},\{0\}^{h+1} \times\{1\}^{k}$ and $U_{j i} \times Y_{i}, V_{j i} \times Y_{i},\{0\}^{h} \times\{1\}^{k}$, respectively, we obtain the claim.

Clearly, $t_{0}^{h} t_{1}^{k} S \in \mathcal{R}^{+}$if and only if $S \in \mathcal{R}^{+}$. Towards a contradiction, assume that $S$ be in $\mathfrak{I} \cap \mathcal{R}^{+}$. By the Claim, there exist $h, k \in \mathbb{N}$ such that $t_{0}^{h} t_{1}^{k} S=$ $\left(S_{Z_{1}}-S_{W_{1}}\right)+\ldots+\left(S_{Z_{m}}-S_{W_{m}}\right)$, for suitable $Z_{i} \approx W_{i}$.

Since $S$ belongs to $\mathcal{R}^{+}$, each $W_{i}$ can be decomposed into pairwise disjoint subsets: $W_{i}=\bigcup_{j=1}^{m} W_{i j}$, in such a way that $W_{i j} \subseteq Z_{j}$. Put $P=(1, \ldots, m)$ and let $\sigma_{1}, \ldots, \sigma_{m}$ be different permutations of $\{1, \ldots, m\}$. Put $X_{i}=\left\{\sigma_{i}(P)\right\} \times Z_{i}$ and 
$Y_{i}=\left\{\sigma_{i}(P)\right\} \times W_{i}$. Then we have $t_{P} \cdot S_{Z_{i}}=S_{X_{i}}$ and $t_{P} \cdot S_{W_{i}}=S_{Y_{i}}$, where $X_{1}, \ldots, X_{m}$ and $Y_{1}, \ldots, Y_{m}$ are pairwise disjoint sets of $\mathbb{W}$.

Put $X=\bigcup_{i=1}^{m} X_{i}$ and $Y=\bigcup_{i=1}^{m} Y_{i}$. Then the series

$$
t_{P} t_{0}^{h} t_{1}^{k} S=\left(S_{X_{1}}-S_{Y_{1}}\right)+\ldots+\left(S_{X_{m}}-S_{Y_{m}}\right)=\left(S_{X}-S_{Y}\right)
$$

is still in $\mathcal{R}^{+} \cap \mathfrak{I}$. By UP we have $Z_{i} \approx X_{i} \approx Y_{i} \approx W_{i}$, and hence $X \approx Y$, by SP.

For each monomial $t^{\mathbf{b}}$ of the series $S_{Y}$ there exists a point $Q \in Y_{i}$ such that $t^{\mathbf{b}}=$ $t_{Q}$. Note that $t_{Q}=t_{P} t_{Q_{i}}$, where $Q_{i}$ is a point in $W_{i}$, so, by hypothesis, $t_{Q_{i}}$ is also a monomial of some series $S_{Z_{j}}$. Hence there exists a permutation of the coordinates of $Q$ such that the corresponding point $Q^{\prime}$ belongs to $X_{j} \subset X$. Repeating this argument for each monomial of the series $S_{Y}$, we can conclude that there exists a transformation $T$ of $Y$, such that $T[Y] \subseteq X$ and $T[Y] \approx Y \approx X$; hence $X=T[Y]$. Then we have $S_{X}-S_{Y}=S_{X}-S_{T[Y]}=0$ and so $\left(S_{X_{1}}-S_{Y_{1}}\right)+\ldots+\left(S_{X_{m}}-S_{Y_{m}}\right)=0$, and this implies $S=0$, absurd. So the proof that $\mathcal{R}^{+} \cap \mathfrak{I}=\emptyset$ is complete.

Given an equinumerosity relation $\approx$, let $\varphi(\approx)$ be the gauge ideal generated by the set $\left\{S_{X}-S_{Y} \mid X \approx Y\right\} \cup\left\{t_{0}-1\right\}$. The map

$$
\varphi:\{\approx \mid \approx \text { equinumerosity relation of } \mathbb{W}\} \rightarrow\{\mathfrak{I} \mid \text { I gauge ideal of } \mathcal{R}\}
$$

is injective. In fact, let $\approx_{1}$ and $\approx_{2}$ be two different equinumerosity relations. Then there exist two sets $X, Y$ of $\mathbb{W}$, such that $X \approx_{1} Y$ and $X \nsim_{2} Y$. Assume without loss of generality that there exists a proper superset $Y^{\prime}$ of $X$ such that $Y^{\prime} \approx_{2} Y$. Put $\varphi\left(\approx_{1}\right)=\mathfrak{I}_{1}$ and $\varphi\left(\approx_{2}\right)=\mathfrak{I}_{2}$; then we have $S_{X}-S_{Y} \in \mathfrak{I}_{1}$ and $S_{Y^{\prime}}-S_{Y} \in \mathfrak{I}_{2}$. If $S_{X}-S_{Y}$ belongs to $\mathfrak{I}_{2}$, then the series $S_{Y^{\prime}}-S_{X}=S_{Y^{\prime} \backslash X}$ is an element of $\mathcal{R}^{+} \cap \mathfrak{I}_{2}$, absurd; so $\mathfrak{I}_{1} \neq \mathfrak{I}_{2}$ and $\varphi$ is 1 -to- 1 .

Given a gauge ideal $\mathfrak{I}$, consider the equinumerosity relation $\approx$ defined by $\mathfrak{I}$ through (**). Putting $\varphi(\approx)=\mathfrak{I}_{\approx}$, we have obviously $\mathfrak{I} \approx \subseteq \mathfrak{I}$, but if there exists $S \in \mathfrak{I} \backslash \mathfrak{I}_{\approx}$, then there exists $P \in \mathcal{R}^{+}$such that either $S+P$ or $S-P$ belongs to $\mathfrak{I}_{\approx}$. Without loss of generality suppose that $S+P \in \mathfrak{I}_{\approx}$; then $S+P \in \mathfrak{I}$ and so $P \in \mathfrak{I}$, absurd. Therefore $\mathfrak{I} \approx=\mathfrak{I}$ and $\varphi$ is biunique.

Having fixed an equinumerosity relation $\approx$ and the corresponding gauge ideal $\mathfrak{I}$, define $j: \mathfrak{N} \rightarrow \mathcal{R} / \mathfrak{I}$ by $j(\mathfrak{n}(X))=S_{X}+\mathfrak{I}$. The application $j$ is well defined, because, whenever $X, Y$ are two equinumerous sets of $\mathbb{W}$, we have $S_{X}-S_{Y} \in \mathfrak{I}$; hence $j(\mathfrak{n}(X))=j(\mathfrak{n}(Y))$. Moreover, if $j(\mathfrak{n}(X))=j(\mathfrak{n}(Y))$, that is, $S_{X}-S_{Y} \in \mathfrak{I}$, then $\mathfrak{n}(X)=\mathfrak{n}(Y)$; hence $j$ is an embedding of $\mathfrak{N}$ into the non-negative part of $\mathcal{R} / \mathfrak{I}$. The range of $j$ is exactly $\left(\mathcal{R}^{+}+\mathfrak{I}\right) / \mathfrak{I}$ because each element of $\mathcal{R}^{+}$is congruent modulo $\mathfrak{I}$ to a characteristic series.

We prove that $j$ induces an ordered semiring structure on $\mathfrak{N}$. Given two disjoint sets $X, Y$ of $\mathbb{W}$, define $\mathfrak{n}(X)+\mathfrak{n}(Y)=j^{-1}(j(\mathfrak{n}(X))+j(\mathfrak{n}(Y)))$; then

$$
\mathfrak{n}(X)+\mathfrak{n}(Y)=j^{-1}\left(S_{X}+S_{Y}+\mathfrak{I}\right)=j^{-1}\left(S_{X \cup Y}+\mathfrak{I}\right)=\mathfrak{n}(X \cup Y) .
$$

Similarly, given two multipliable sets $X, Y$ of $\mathbb{W}$, define

$$
\mathfrak{n}(X) \cdot \mathfrak{n}(Y)=j^{-1}(j(\mathfrak{n}(X)) \cdot j(\mathfrak{n}(Y)))
$$

then

$$
\mathfrak{n}(X) \cdot \mathfrak{n}(Y)=j^{-1}\left(S_{X} \cdot S_{Y}+\mathfrak{I}\right)=j^{-1}\left(S_{X \times Y}+\mathfrak{I}\right)=\mathfrak{n}(X \times Y) .
$$

The sum and product are well defined by Proposition 1.3 .

If $X, Y \in \mathbb{W}$ are not equinumerous, i.e. $S_{X}-S_{Y} \in \mathcal{R} \backslash \mathfrak{I}$, then there exists a set $Z \in \mathbb{W}$ such that either $S_{X}-S_{Y}+S_{Z}$ or $S_{X}-S_{Y}-S_{Z}$ belongs to $\mathfrak{I}$, by the third 
property of gauge ideals. So define a total order $<$ on $\mathfrak{N}$ by putting

$$
\mathfrak{n}(X)<\mathfrak{n}(Y) \Longleftrightarrow \exists Z \in \mathbb{W}, Z \neq \emptyset \text {, such that } S_{X}-S_{Y}+S_{Z} \in \mathfrak{I} .
$$

Note that, by Lemma 1.11, if $\mathfrak{n}(X)<\mathfrak{n}(Y)$ we can always choose the set $Z$ so that $X$ and $Z$ are disjoint and hence $S_{X}-S_{Y}+S_{Z}=S_{X \cup Z}-S_{Y} \in \mathfrak{I}$. Therefore $\mathfrak{n}(X)<\mathfrak{n}(Y)$ holds if and only if there exists a proper superset $Y^{\prime}$ of $X$ such that $Y^{\prime} \approx Y$. By Proposition 1.4 we conclude that the relation $<$ is a total order on $\mathfrak{N}$ and hence $(\mathfrak{N},+, \cdot,<)$ is an ordered semiring.

Remark 1.14. In [9] a class of particular equinumerosity relations has been considered, namely those which are preserved under "natural transformations", i.e. bijections that preserve the support (set of components) of each tuple.

Let us call an equinumerosity relation natural if it satisfies the following Natural Transformation Principle, which is a severe strengthening of TP:

(NP) If $T$ is 1 -to- 1 on $X \in \mathbb{W}$ and $\operatorname{supp}(T(x))=\operatorname{supp}(x)$ for all $x \in X$, then $X \approx T[X]$.

For $S \in \mathcal{R}$ let $S^{\prime}$ be the associated squarefree series, i.e. the series obtained by replacing each monomial in $S$ by the corresponding squarefree monomial and summing up the corresponding coefficients. I.e.

$$
\text { if } S=\sum_{\mathbf{a} \in \mathbf{A}} n_{\mathbf{a}} t^{\mathbf{a}}, \text { then } S^{\prime}=\sum_{F \in \mathbb{N}<\omega}\left(\sum_{\operatorname{supp}(\mathbf{a})=F} n_{\mathbf{a}}\right) t_{F},
$$

where

$$
\operatorname{supp}(\mathbf{a})=\left\{n \in \mathbb{N} \mid a_{n} \neq 0\right\} \text { and } t_{F}=\prod_{n \in F} t_{n} .
$$

Let $\mathfrak{I}_{1}$ be the kernel of the map $S \mapsto S^{\prime}$, i.e. the ideal of $\mathcal{R}$ generated by the set $\left\{S-S^{\prime} \mid S \in \mathcal{R}^{+}\right\}$. Then we have

Proposition 1.15. The equinumerosity relation $\approx$ is natural if and only if the corresponding gauge ideal $\mathfrak{I}$ includes $\mathfrak{I}_{1}$.

Proof. Let $\mathfrak{I}$ be a gauge ideal that includes $\mathfrak{I}_{1}$. We prove that the equivalence relation defined by the relation

$$
X \approx Y \Longleftrightarrow S_{X}-S_{Y} \in \mathfrak{I}
$$

is a natural equinumerosity. The principles AP, ZP, UP, PP hold because the ideal $\mathfrak{I}$ is gauge. Hence we have only to prove that if $T$ is a natural transformation of a set $X \in \mathbb{W}$ then $X \approx T[X]$, or equivalently $S_{X}-S_{T[X]} \in \mathfrak{I}$.

Let $S_{X}^{\prime}$ and $S_{T[X]}^{\prime}$ be the squarefree series associated to the series $S_{X}$ and $S_{T[X]}$, respectively. We prove that $S_{X}^{\prime}=S_{T[X]}^{\prime}$, so the series $S_{X}-S_{T[X]}=S_{X}-S_{X}^{\prime}+$ $S_{T[X]}^{\prime}-S_{T[X]}$ belongs to $\mathfrak{I}_{1} \subseteq \mathfrak{I}$. Let $n_{\mathbf{a}} t^{\text {a }}$ be a (squarefree) monomial of the series $S_{X}^{\prime}$; then there exist distinct points $P_{1}, \ldots, P_{n_{\mathrm{a}}}$ of the set $X$ that produce the monomial $t^{\mathbf{a}}$. Now consider the points $T\left(P_{1}\right), \ldots, T\left(P_{n_{\mathbf{a}}}\right)$ that belong to the set $T[X]$. The map $T$ preserves the support of each point, hence in the series $S_{T[X]}^{\prime}$ the monomial $t^{\mathbf{a}}$ appears with a coefficent equal to $n_{\mathbf{a}}$ because all and only $T\left(P_{1}\right), \ldots, T\left(P_{n_{\mathbf{a}}}\right)$ can have this support. So we conclude that $S_{T[X]}^{\prime}=S_{X}^{\prime}$.

Conversely, given a natural equinumerosity $\approx$ over $\mathbb{W}$, let $\mathfrak{I}$ be the ideal generated by the set $\left\{S_{X}-S_{Y} \mid X \approx Y\right\} \cup\left\{t_{0}-1\right\}$. The ideal $\mathfrak{I}$ is gauge, because $\approx$ is an equinumerosity relation, so we need only to prove that $\mathfrak{I}$ includes the ideal $\mathfrak{I}_{1}$ generated by the set $\left\{S-S^{\prime} \mid S \in \mathcal{R}^{+}\right\}$. 
Let $S$ be a series of $\mathcal{R}^{+}$, and pick (not necessarily distinct) sets $X_{1}, \ldots, X_{n} \in \mathbb{W}$ such that $S=a+S_{X_{1}}+\ldots+S_{X_{n}}$, taking care that, in each set $X_{i}$, the tuples of each support of size $k$ be at most $k$ !. Then each squarefree series $S_{X_{i}}^{\prime}$ is equal to the characteristic series of a set $Y_{i} \in \mathbb{W}$, and we can define natural transformations $T_{i}: X_{i} \rightarrow Y_{i}$ in such a way that $T_{i}(x)$ is a permutation of the support of $x$, for all $x \in X_{i}$. Hence

$$
S^{\prime}=a+S_{X_{1}}^{\prime}+\ldots+S_{X_{n}}^{\prime}=a+S_{Y_{1}}+\ldots+S_{Y_{n}}=a+S_{T_{1}\left[X_{1}\right]}+\ldots+S_{T_{n}\left[X_{n}\right]},
$$

and we conclude that the series $S-S^{\prime}$ belongs to $\mathfrak{I}$.

The very same proof of Theorem 1.13 can be used to prove that there exists a biunique correspondence between natural equinumerosities and gauge ideals $\mathfrak{I}$ including $\mathfrak{I}_{1}$, and that there exists a unique order preserving embedding $j$ of the set $\mathfrak{N}$ of the natural numerosities corresponding to the ideal $\mathfrak{I}$ onto the non-negative part of $\mathcal{R} / \mathfrak{I}$. In the next section we shall give a complete characterization of all natural numerosities as hypernatural numbers from suitable ultrapowers of $\mathbb{N}$.

\section{Equinumerosities through Ultrafilters}

We give in this section a construction of equinumerosity relations through suitable ultrafilters.

Definition 2.1. Let $\mathbb{X}$ be the set of all sequences of non-negative real numbers $\mathbf{x}=\left\langle x_{0}, \ldots, x_{n}, \ldots\right\rangle$ such that the series $\sum x_{n}=\|\mathbf{x}\|$ converges.

For $S \in \mathcal{R}$ let $S(\mathbf{x})$ be the value taken by $S$ when $x_{n}$ is assigned to the variable $t_{n}$. (So e.g. $\|\mathbf{x}\|^{d}=S_{\mathbb{N}^{d}}(\mathbf{x})$.)

- A subset $\mathbb{I} \subseteq \mathbb{X}$ is a counting set (of assignments) if for all $k \in \mathbb{N}$ the set $\mathbb{I}_{k}=\left\{\mathbf{i} \in \mathbb{I} \mid i_{0}=i_{1}=\ldots=i_{k}=1\right\} \neq \emptyset$;

- an ultrafilter $\mathcal{U}$ on the counting set $\mathbb{I}$ is suitable for $\mathbb{I}$ if for all $k \in \mathbb{N}$ the set $\mathbb{I}_{k}$ is in $\mathcal{U}$;

- the counting set $\mathbb{I}$ is suitable for the ideal $\mathfrak{I}$ of $\mathcal{R}$ if for all $S \in \mathfrak{I}$ and all $k \in \mathbb{N}$ there exists $\mathbf{i} \in \mathbb{I}_{k}$ such that $S(\mathbf{i})=0$.

Let $\mathbb{I}$ be a counting set, and define the counting map

$$
\Phi: \mathcal{R} \rightarrow \mathbb{R}^{\mathbb{I}} \text { by } \Phi(S)=\langle S(\mathbf{i})\rangle_{\mathbf{i} \in \mathbb{I}} .
$$

Then $\Phi$ is a ring homomorphism that preserves the respective partial orderings.

If $\mathcal{U}$ is an ultrafilter suitable for $\mathbb{I}$ and $\pi_{\mathcal{U}}: \mathbb{R}^{\mathbb{I}} \rightarrow \mathbb{R}_{\mathcal{U}}^{\mathbb{I}}$ is the natural projection onto the corresponding ultrapower, put $\phi_{\mathcal{U}}=\pi_{\mathcal{U}} \circ \Phi$, so that

$$
\phi_{\mathcal{U}}(S)=\left[\langle S(\mathbf{i})\rangle_{\mathbf{i} \in \mathbb{I}}\right] \mathcal{U} .
$$

Then $\phi_{\mathcal{U}}$ is a ring homomorphism whose kernel $\mathfrak{I}=\operatorname{ker} \phi_{\mathcal{U}}$ is a prime ideal of $\mathcal{R}$ that includes $\mathfrak{I}_{0}$ and is disjoint from $\mathcal{R}^{+}$. Moreover, the counting set $\mathbb{I}$ turns out to be suitable for the ideal $\mathfrak{I}$.

The map $X \mapsto \phi_{\mathcal{U}}\left(S_{X}\right)$ induces an equivalence relation between point sets

$$
X \approx_{\mathcal{U}} Y \Longleftrightarrow\left\{\mathbf{i} \in \mathbb{I} \mid S_{X}(\mathbf{i})=S_{Y}(\mathbf{i})\right\} \in \mathcal{U}
$$

that satisfies all conditions of an equinumerosity relation but possibly Zermelo's Principle ZP.

If the kernel $\mathfrak{I}=\operatorname{ker} \phi_{\mathcal{U}}$ is a gauge ideal, then $\approx_{\mathcal{U}}$ is an equinumerosity relation whose set of numerosities is (isomorphic to) a discrete semiring of hyperreal 
numbers, namely a subsemiring of the non-negative part of the ultrapower $\mathbb{R}_{\mathcal{U}}^{\mathbb{I}}$. A simple rephrasing of the definition gives

Proposition 2.2. The equivalence $\approx_{\mathcal{U}}$ is an equinumerosity, or equivalently $\mathfrak{I}=$ $\operatorname{ker} \phi_{\mathcal{U}}$ is a gauge ideal of $\mathcal{R}$, if and only if, for all $S \in \mathcal{R}$,

$$
\{\mathbf{i} \in \mathbb{I} \mid S(\mathbf{i})>0\} \in \mathcal{U} \Longleftrightarrow \exists P \in \mathcal{R}^{+} \text {s.t. }\{\mathbf{i} \in \mathbb{I} \mid S(\mathbf{i})=P(\mathbf{i})\} \in \mathcal{U} .
$$

Conversely we can state

Theorem 2.3. Let $\approx$ be an equinumerosity, and let $\mathfrak{I}$ be the corresponding gauge ideal of $\mathcal{R}$. Assume that there exists a counting set $\mathbb{I}$ suitable for $\mathfrak{I}$. Then there exists an ultrafilter $\mathcal{U}$ suitable for $\mathbb{I}$ such that the equinumerosity $\approx$ coincides with the equivalence $\approx_{\mathcal{U}}$ induced by the counting map $\phi_{\mathcal{U}}$.

So the set of numerosities $\mathfrak{N}$ of $\approx$ is isomorphic to a discrete subsemiring of the non-negative part of the ultrapower $\mathbb{R}_{\mathcal{U}}^{\mathbb{I}}$.

Proof. We first of all remark that the family $\mathcal{F}$ of the zero-sets $Z(S)=\{\mathbf{i} \in \mathbb{I} \mid S(\mathbf{i})$ $=0\}$ for $S \in \mathfrak{I}$ has the finite intersection property, because $Z(S) \cap Z(T)=$ $Z\left(S^{2}+T^{2}\right)$. Let $\mathcal{U}$ be any ultrafilter containing $\mathcal{F}$. We claim that if $Z(S) \in \mathcal{U}$, then $S \in \mathfrak{I}$.

If $S \notin \mathfrak{I}$ we may assume without loss of generality that there exists $P \in \mathcal{R}^{+}$and $I \in \mathfrak{I}$ such that $S=I+P$. Then $Z(S) \cap Z(I) \subseteq Z(P)$, and $Z(P) \cap \mathbb{I}_{k}=\emptyset$ for every sufficiently large $k$. But $\mathbb{I}_{k}$ is the intersection of the zero-sets of the polynomials $t_{n}-1$ for $n \leq k$, so it belongs to $\mathcal{U}$, a contradiction. Hence $\mathfrak{I}=\operatorname{ker} \phi_{\mathcal{U}}$, and the thesis follows.

In the case of natural equinumerosities, one can consider only 0-1 assignments, because only these annihilate the series

$$
\sum_{n \in \mathbb{N}}\left(t_{n}^{2}-t_{n}\right)^{2}
$$

Namely, arrange the set of all eventually zero sequences of zeroes and ones in a sequence $\mathbb{L}=\left\langle\mathbf{x}_{F} \mid F \in \mathbb{N}<\omega\right\rangle$, where $\mathbf{x}_{F}(n)=1$ if and only if $n \in F$. Then

Lemma 2.4. The counting map $\Phi: \mathcal{R} \rightarrow \mathbb{Z}^{\mathbb{L}}$ such that $\Phi(S)=\left\langle S\left(\mathbf{x}_{F}\right) \mid F \in \mathbb{N}<\omega\right\rangle$ is a surjective homomorphism of partially ordered rings whose kernel is the ideal $\mathfrak{I}_{1}$ that consists of all those series whose corresponding squarefree series is 0 .

Proof. The map $\Phi$ is a homomorphism by definition, and clearly it maps $\mathcal{R}^{+}$into $\mathbb{N}^{\mathbb{L}}$.

Given $S=\sum n_{\mathbf{a}} t^{\mathbf{a}} \in \mathcal{R}$ and $F \in \mathbb{N}<\omega$, recall that $\operatorname{supp}(\mathbf{a})=\left\{n \in \mathbb{N} \mid a_{n} \neq\right.$ $0\}$ and $t_{F}=\prod_{n \in F} t_{n}$. Put $n_{F}=\sum_{F=\operatorname{supp}(\mathbf{a})} n_{\mathbf{a}}$; then $S\left(\mathbf{x}_{F}\right)=\sum_{E \subseteq F} n_{E}$, and $S^{\prime}=\sum_{F \in \mathbb{N}<\omega} n_{F} t_{F}$. By the inclusion-exclusion principle one has $n_{F}=$ $\sum_{E \subseteq F}(-1)^{|F \backslash E|} S\left(\mathbf{x}_{E}\right)$. Hence $S \in \operatorname{ker} \Phi$ if and only if $n_{F}=0$ for all $F \in \mathbb{N}<\omega$, or equivalently $S^{\prime}=0$. In particular, no non-zero squarefree series lies in the kernel of $\Phi$.

On the other hand, given $g \in \mathbb{Z}^{\mathbb{L}}$ one has that any series $S=\sum n_{\mathbf{a}} t^{\mathbf{a}}$ such that $n_{F}=\sum_{E \subseteq F}(-1)^{|F \backslash E|} g(E)$ satisfies $\Phi(S)=g$. So $\left|n_{F}\right| \leq \sum_{E \subset F}|g(E)|$. Put $d_{n}=\sum_{E \subseteq\{0, \ldots, n\}}|g(E)|$; then we can find in $\mathcal{R}$ a series $S$ of degree not exceeding $d_{n}$ in each variable $t_{n}$ that satisfies the condition above. 
We are now ready to classify all natural equinumerosity relations. Call gauge a fine 4 ultrafilter $\mathcal{U}$ over $\mathbb{N}<\omega$ if every square $f^{2} \in \mathbb{N}^{\mathbb{L}}$ is equivalent modulo $\mathcal{U}$ to a function $g$ such that $n_{F}=\sum_{E \subseteq F}(-1)^{|F \backslash E|} g(E) \geq 0$ for all $F \in \mathbb{N}<\omega$. (Remark that $\Phi\left(\sum n_{F} t_{F}\right)=g$. $)$

Theorem 2.5. Let $\approx$ be a natural equinumerosity, and let $\mathfrak{I}$ be the corresponding gauge ideal of $\mathcal{R}$. Then $\mathbb{L}$ is suitable for $\mathfrak{I}$, and there exists a unique gauge ultrafilter $\mathcal{U}$ over $\mathbb{N}<\omega$ suitable for $\mathbb{L}$ such that

$$
X \approx Y \Longleftrightarrow\left\{F \in \mathbb{N}^{<\omega} \mid S_{X}\left(\mathbf{x}_{F}\right)=S_{Y}\left(\mathbf{x}_{F}\right)\right\} \in \mathcal{U}
$$

Hence the counting map $\Phi$ induces an ordered semiring isomorphism between the set of numerosities $\mathfrak{N}$ of $\approx$ and the ultrapower $\mathbb{N}_{\mathcal{U}}^{\mathbb{N}}$.

Conversely, the condition (***) defines a natural equinumerosity on $\mathbb{W}$ if and only if the ultrafilter $\mathcal{U}$ is gauge.

Proof. The ideal $\mathfrak{I}$ includes the ideal $\mathfrak{I}_{1}$, hence in particular all series whose corresponding squarefree is 0 , i.e. the kernel of $\Phi$. Let $\mathfrak{J}=\Phi[\mathfrak{I}]$ be the ideal of $\mathbb{Z}^{\mathbb{L}}$ corresponding to $\mathfrak{I}$. Then $\mathcal{R} / \mathfrak{I}$ is isomorphic to $\mathbb{Z}^{\mathbb{L}} / \mathfrak{J}$, and every element of $\mathfrak{J}$ has some component equal to 0 . In fact, assume that $f \in \mathfrak{J}$ has no zeroes, and multiply $f$ by a suitable sequence of \pm 1 so as to obtain a sequence $g \in \mathfrak{J}$ of positive integers. Then $g$ can be written as

$$
g=1+h_{1}^{2}+h_{2}^{2}+h_{3}^{2}+h_{4}^{2}=\Phi\left(1+S_{1}^{2}+S_{2}^{2}+S_{3}^{2}+S_{4}^{2}\right) .
$$

But the series $1+S_{1}^{2}+S_{2}^{2}+S_{3}^{2}+S_{4}^{2}$ is equivalent modulo $\mathfrak{I}$ to an element of $\mathcal{R}^{+}$, and so its image $g$ under $\Phi$ cannot belong to $\mathfrak{J}$. So the counting set $\mathbb{L}$ is suitable for $\mathfrak{I}$, and $\mathfrak{J}$ is a prime zero-ideal that is determined by an ultrafilter $\mathcal{U}$ over $\mathbb{L}$, which in turn is suitable for $\mathbb{L}$, because $\mathbb{I}_{k}$ is the intersection of the zero-sets of the polynomials $t_{n}-1$ for $0 \leq n \leq k$. It follows that $\mathcal{U}$ is a fine ultrafilter and that condition (***) holds.

The ultrafilter $\mathcal{U}$ is gauge, because, given $f^{2}=\Phi\left(S^{2}\right)>0$, there exists $P \in \mathcal{R}^{+}$ that is equivalent to $S^{2}$ modulo $\mathfrak{I}$. Put $\Phi(P)=g$; then clearly $g \equiv f^{2}$ modulo $\mathcal{U}$ and satisfies the equalities $\sum_{E \subset F}(-1)^{|F \backslash E|} g(E) \geq 0$ because $P \in \mathcal{R}^{+}$.

Conversely, if $\mathcal{U}$ is a gauge ultrafilter, then (***) defines an equinumerosity relation by Proposition 2.2, which is natural because the corresponding ideal of $\mathcal{R}$ includes $\mathfrak{I}_{1}$.

In particular, we have

Corollary 2.6. Condition $\stackrel{* * * *}{\longrightarrow}$ provides a biunique correspondence between natural equinumerosity relations $\approx$ on $\mathbb{W}$ and gauge ultrafilters $\mathcal{U}$ on $\mathbb{N}<\omega$ in such a way that the following diagram commutes:

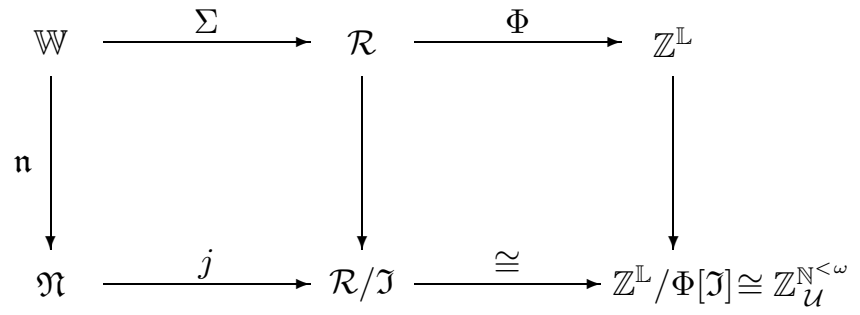

${ }^{4}$ The ultrafilter $\mathcal{U}$ is fine if all cones $C_{n}=\{F \mid n \in F\}$ belong to $\mathcal{U}$.

${ }^{5}$ Recall that every element of $\mathbb{N}^{\mathbb{L}}$ is a sum of squares. 
In particular all sets of natural numerosities can be taken to be sets of hypernatural numbers of the corresponding ultrapowers $\mathbb{N}_{\mathcal{U}}^{<<}$.

Remark 2.7. Put $F_{k}=\{0, \ldots, k\}$. In this context, the asymptotic numerosities defined in [5] can be characterized as the restrictions to $\mathbb{W}_{0}$ of those natural equinumerosities for which $\mathbb{I}=\left\{\mathbf{x}_{F_{k}} \mid k \in \mathbb{N}\right\}$ is a suitable counting set.

\section{Final Remarks And open Questions}

It is interesting to remark that the natural numerosities satisfy a "Finite Approximation Principle" analogous to that considered in [2]. Let $\mathfrak{n}$ be a natural numerosity function, and for $X \in \mathbb{W}$ and $F \in \mathbb{N}<\omega$ put $X_{F}=X \cap \bigcup_{n \in \mathbb{N}} F^{n}$ so that $S_{X}\left(\mathbf{x}_{F}\right)=\left|X_{F}\right|$. Then

(FAP) If $\left|X_{F}\right| \leq\left|Y_{F}\right|$ for all $F \in \mathbb{N}^{<\omega}$, then $\mathfrak{n}(X) \leq \mathfrak{n}(Y)$.

In fact, if $\mathcal{U}$ is the gauge ultrafilter corresponding to the numerosity $\mathfrak{n}$, we have

$$
\mathfrak{n}(X) \leq \mathfrak{n}(Y) \Longleftrightarrow\left\{F \in \mathbb{N}^{<\omega}|| X_{F}|\leq| Y_{F} \mid\right\} \in \mathcal{U} .
$$

This fact suggests a "Cantorian" characterization of natural equinumerosity by means of a class of particular bijections, similar to the one obtained in [5] for asymptotic equinumerosity. Call a $\mathcal{U}$-congruence between $X$ and $Y$ a 1-to-1 map $\tau: X \rightarrow Y$ such that $\left\{F \in \mathbb{N}<\omega \mid \tau\left[X_{F}\right]=Y_{F}\right\} \in \mathcal{U}$. Then we have

Remark 3.1. Let $\approx$ be a natural equinumerosity, and let $\mathcal{U}$ be the corresponding gauge ultrafilter over $\mathbb{N}<\omega$. Then, by definition,

if there exists a $\mathcal{U}$-congruence between $X$ and $Y$, then $X \approx Y$.

The reverse implication seems difficult to prove in general. A simple inductive definition of $\tau$ can be given whenever the ultrafilter $\mathcal{U}$ contains a chain. But in this case the equinumerosity becomes asymptotic after an appropriate reordering of $\mathbb{N}$.

When the ultrafilter is Ramsey the situation is much simpler, namely

Corollary 3.2. A fine Ramsey ultrafilter $\mathcal{U}$ on $\mathbb{N}<\omega$ provides a natural equinumerosity relation on $\mathbb{W}$. The corresponding set of numerosities $\mathbb{N}^{\mathbb{N}}<\omega$ is isomorphic to the ultrapower $\mathbb{N}_{\sigma \mathcal{U}}^{\mathbb{N}}$, where $\sigma: \mathbb{N}^{<\omega} \rightarrow \mathbb{N}$ is defined by $\sigma(F)=|F|$. This equinumerosity is characterized by the class of the $\mathcal{U}$-congruences and becomes asymptotic after a suitable reordering of $\mathbb{N}$.

Proof. Consider the coloring of $[\mathbb{N}<\omega]^{2}$ given by $c(\{F, G\})=0$ if $F$ is comparable with $G$ and $c(\{F, G\})=1$ otherwise. The ultrafilter $\mathcal{U}$ being Ramsey, it contains a homogeneous subset $H$, which is a chain, because $\mathcal{U}$ is fine.

In order to prove the first assertion, we show that $\mathcal{U}$ is gauge. Remark that, $\mathcal{U}$ being Ramsey, any non-negative function $f: \mathbb{N}<\omega \rightarrow \mathbb{Z}$ is non-decreasing on some subchain $H_{0} \subset H_{1} \subset \ldots \subset H_{n} \subset \ldots$ of $H$ that belongs to $\mathcal{U}$. Define the series

$$
S=f\left(H_{0}\right) t_{H_{0}}+\sum_{n \in \mathbb{N}}\left(f\left(H_{n+1}\right)-f\left(H_{n}\right)\right) t_{H_{n+1}} \in \mathcal{R}^{+} .
$$

Then $\Phi(S)$ is equivalent to $f$ modulo $\mathcal{U}$, and so $\mathcal{U}$ is gauge.

Assume without loss of generality that $H$ is complete, i.e. that the map $\sigma$ restricted to $H$ is onto $\mathbb{N}$. Then $\sigma$ induces an isomorphism between the ultrapowers $\mathbb{Z}_{\mathcal{U}}^{\mathbb{N}<\omega}$ and $\mathbb{Z}_{\sigma \mathcal{U}}^{\mathbb{N}}$, and the second assertion is proved.

Given equinumerous sets $X$ and $Y$, let $H_{0} \subset H_{1} \subset \ldots \subset H_{n} \subset \ldots$ be a subchain of $H$ such that $\left|X_{H_{n}}\right|=\left|Y_{H_{n}}\right|$ for all $n \in \mathbb{N}$. Define $\tau: X \rightarrow Y$ by gluing together 
disjoint bijections $\tau_{0}: X_{H_{0}} \rightarrow Y_{H_{0}}$ and $\tau_{n+1}: X_{H_{n+1}} \backslash X_{H_{n}} \rightarrow Y_{H_{n+1}} \backslash Y_{H_{n}}$ for all $n \in \mathbb{N}$. Then $\tau$ is a $\mathcal{U}$-congruence between $X$ and $Y$.

Finally, any complete chain $H$ in $\mathcal{U}$ provides a reordering of $\mathbb{N}$ such that $H_{k}$ becomes $F_{k}$. Hence, with respect to this reordering, the equinumerosity is asymptotic.

Remark that the ultrafilter $\sigma \mathcal{U}$ on $\mathbb{N}$ defined above is Ramsey whenever the gauge ultrafilter $\mathcal{U}$ contains a (complete) chain, because every non-negative function is non-decreasing modulo $\sigma \mathcal{U}$. So natural equinumerosities exist under mild set theoretic hypotheses, namely those that provide Ramsey ultrafilters over $\mathbb{N}$, and the corresponding numerosities are, up to isomorphism, the asymptotic numerosities of [5]. On the other hand, Andreas Blass has recently proved that there exist gauge ultrafilters that are not $P$-points whenever there are infinitely many pairwise nonisomorphic Ramsey ultrafilters on $\mathbb{N} 6$ Namely, let $\mathcal{V}$ and $\left\{\mathcal{U}_{n} \mid n \in \mathbb{N}\right\}$ be pairwise non-isomorphic Ramsey ultrafilters on $\mathbb{N}$, let $\left\{A_{n} \mid n \in \mathbb{N}\right\}$ be a partition of $\mathbb{N}$ with $A_{n} \in \mathcal{U}_{n}$, and let $\mathcal{U}$ be the " $\mathcal{V}$-sum" ultrafilter such that $U \in \mathcal{U}$ if and only if $\left\{n \in \mathbb{N} \mid U \cap A_{n} \in \mathcal{U}_{n}\right\} \in \mathcal{V}$. If $a$ is the $m$ th element of $A_{n}$, let $\varphi(a) \in \mathbb{N}<\omega$ be the set containing the first $m$ elements of the sets $A_{i}$ for $i \leq n$. Then the ultrafilter $\varphi \mathcal{U}$ is gauge and isomorphic to $\mathcal{U}$, and hence not a $P$-point, because the map $\varphi: \mathbb{N} \rightarrow \mathbb{N}<\omega$ is injective.

So natural numerosities that are not isomorphic to asymptotic numerosities exist under any hypothesis yielding a sequence of pairwise non-isomorphic Ramsey ultrafilters, e.g. the Continuum Hypothesis or Martin's Axiom. Actually, we conjecture that Rudin-Keisler below any gauge ultrafilters there are always P-points, and in this case the most interesting question, namely that of the existence of natural equinumerosities in ZFC alone, would be solved in the negative. Combined with the "geometric" intuition that the size of the diagonal might be different from that of the side, this conjecture is the reason why we did not include the Natural Transformation Principle NP in the definition of equinumerosity. On the other hand, the existence of gauge ideals of the ring $\mathcal{R}$ seems to be weaker than that of gauge ultrafilters, and so the existence of (non-natural) equinumerosities might be provable in ZFC. However, this question remains open for now.

\section{REFERENCES}

[1] Vieri Benci and Mauro Di Nasso, Numerosities of labelled sets: a new way of counting, Adv. Math. 173 (2003), no. 1, 50-67, DOI 10.1016/S0001-8708(02)00012-9. MR.1954455 (2004b:03065)

[2] Vieri Benci, Mauro Di Nasso, and Marco Forti, An Aristotelian notion of size, Ann. Pure Appl. Logic 143 (2006), no. 1-3, 43-53, DOI 10.1016/j.apal.2006.01.008. MR2258620(2007g:03070)

[3] Vieri Benci, Marco Forti, and Mauro Di Nasso, The eightfold path to nonstandard analysis, Nonstandard methods and applications in mathematics, Lect. Notes Log., vol. 25, Assoc. Symbol. Logic, La Jolla, CA, 2006, pp. 3-44. MR2209073 (2006m:26066)

[4] Vieri Benci, Mauro Di Nasso, and Marco Forti, An Euclidean measure of size for mathematical universes, Logique et Anal. (N.S.) 50 (2007), no. 197, 43-62. MR2308603 (2008d:03057)

[5] Andreas Blass, Mauro Di Nasso, and Marco Forti, Quasi-selective ultrafilters and asymptotic numerosities, Adv. Math. 231 (2012), no. 3-4, 1462-1486, DOI 10.1016/j.aim.2012.06.021. MR2964612

[6] C. C. Chang and H. J. Keisler, Model theory, 3rd ed., Studies in Logic and the Foundations of Mathematics, vol. 73, North-Holland Publishing Co., Amsterdam, 1990. MR 1059055 (91c:03026)

\footnotetext{
6 Personal communication to the first author, September 3, 2012.
} 
[7] Mauro Di Nasso and Marco Forti, Numerosities of point sets over the real line, Trans. Amer. Math. Soc. 362 (2010), no. 10, 5355-5371, DOI 10.1090/S0002-9947-2010-04919-0. MR2657683 (2011i:03059)

[8] Euclid, The thirteen books of Euclid's Elements translated from the text of Heiberg. Vol. I: Introduction and Books I, II. Vol. II: Books III-IX. Vol. III: Books X-XIII and Appendix, Dover Publications Inc., New York, 1956. Translated with introduction and commentary by Thomas L. Heath; 2nd ed. MR0075873 (17,814b)

[9] G. Morana Roccasalvo, Numerosità di sottoinsiemi di $\mathbb{N}^{k}$ (Italian). Tesi di Laurea, Pisa 2012.

Dipartimento di Matematica, University of Pisa, Via Buonarroti 1C, 56100 Pisa, Italy E-mail address: forti@dma.unipi.it

Dipartimento di Matematica, University of Pisa, Via Buonarroti 1C, 56100 Pisa, Italy E-mail address: moranaroccasalvo@mail.dm.unipi.it 